\title{
Coverage of Femoral Head at Weight-Bearing Interface of the Hip Joint in Children: An MRI Analysis
}

\author{
Si-Hui Zeng ${ }^{1 *}$, Hong-Sheng Liu ${ }^{1}$, Hong-Wen $\mathrm{Xu}^{2}$, Yuan-Kai Chen ${ }^{1}$, \\ Ding-Qiang Miao ${ }^{1}$, Li-Yi Nie $^{1}$ \\ ${ }^{1}$ Imaging Center, Guangzhou, China \\ ${ }^{2}$ Department of Orthopedics, Guangzhou Women and Children's Medical Center, Guangzhou, China \\ Email: * stephaniezeng@163.com
}

Received April 14, 2013; revised May 14, 2013; accepted May 28, 2013

Copyright (C) 2013 Si-Hui Zeng et al. This is an open access article distributed under the Creative Commons Attribution License, which permits unrestricted use, distribution, and reproduction in any medium, provided the original work is properly cited.

\begin{abstract}
Objective: To analyze the features of coverage of femoral head at weight-bearing interface of the hip joints in children. Material and Methods: MRI scans of the hips were performed in 95 normal children aged from 1 to 8 years. Radial scans of the hip joints were performed using FFE sequence. Review the morphological features of weight-bearing interface of the acetabulum and the femoral head. Total covering angle (TCA), acetabular covering angle (ACA) and labral covering angle (LCA) were measured, inter-group comparison and correlation analysis were done. Result: The acetabulum and the femoral head had congruent articulating surface at each weight-bearing position. There was no statistical TCA difference at each position. Average ACA increased, while average LCA decreased from anterior to posterior. TCA correlated with LCA, ACA negatively correlated with LCA. Conclusion: TCA is a good index in indicating stability of the hip joint. Cartilage ossifies slower at posterior than anterior positions. Cartilage acetabulum and the labrum serve as complementary structures that contribute in total stabilizing of the hip joint in development.
\end{abstract}

Keywords: Magnetic Resonance; Hip Joint; Weight-Bearing; Children; Coverage of the Femoral Head

\section{Introduction}

The hip joint is the largest weight-bearing joint in the human being. The normal development of a child's hip is the result of synchronized growing of acetabulum and femoral head [1]. Conventional radiography and computed tomography have long been the major imaging techniques in diagnosing developmental hip diseases $[2,3]$. The most common diseases that affect a child's hip are developmental dysplasia of the hip, Legg-CalvePerthes, and infections. However, radiological examination is limited in visualizing articular cartilage, which cannot perfectly reflect anatomical characteristics in accordance with physiological status. There have been several MR studies on development of the hip joint in recent years. MR is proved to be a good modality in imaging the hip joint for its better muscular-skeletal resolution. Application of radial scanning is popular in reviewing the interacting surface of the joint [4-6]. Measuring degree of coverage of femoral head by acetabulum and labrum at weight-bearing area can reflect the physiological status of the hip joint. However, there were few MR studies on

"Corresponding author. development of hip joint in children. The purpose of this study was to measure the degree of coverage of femoral head at weight-bearing interface by radial MRI and evaluate the developmental characteristics of the hip joint.

\section{Materials and Methods}

\subsection{Subjects}

This was a prospective study of MR image from healthy volunteers and patients who were referred for pelvic MRI for some other reasons but without suspicious hip joint disease from April 2010 to December 2012 in Guangzhou Women and Children's hospital. Children were given routine physical examination from experienced orthopedists to exclude any hip disease. Those with family history of skeletal malformations or positive MR findings were also excluded. The study had Ethics Committee's approval. Informed consent from the child's guardian was required. 95 children were recruited and 190 hips were imaged. There were 48 boys and 47 girls respectively, aging 1 - 8 years old, and the median age was 61 months. 


\subsection{MR Imaging}

MR of hip joint was performed on a 1.5-T superconducting MR scanner (Achieva Release 2.6.3.7, The Netherlands) using a 4 element phased-array SENSE-Body coil. Children were placed supine, feet put close together. Axial fat-suppressed T2-weighted images of the hip joint was obtained first, coronal FFE images through the center of femoral head was obtained next, in order to have a overview of the hip joint. A scout view including the full circumference of the acetabular margin was corrected for acetabular anteversion in two-steps: 1) position the orienting line along anterior and posterior margin of the acetabulum on axial slice of the center of femoral head (Figure 1); 2) position the orienting line along upper and lower margin of the acetabulum on coronal slice of the center of femoral head (Figure 2). Oblique fat-suppressed T2-weighted images of the acetabulum were generated as a scout view (Figure 3). Radial FFE images were obtained at $15^{\circ}$ intervals on center of the femoral head in the scout view. Scanning protocols were shown at Table 1.

\subsection{Evaluation Items and Statistical Analysis}

Morphology of bony acetabulum, articular cartilage and labrum were reviewed. Those cases with distinctive anatomical margin which allowed precise line drawing for measurement were included in statistical analysis. The coverage of femoral head by the acetabulum and la brum was measured at 5 positions from the superior quarter of the acetabulum. These positions were anterosuperior $30^{\circ}$,

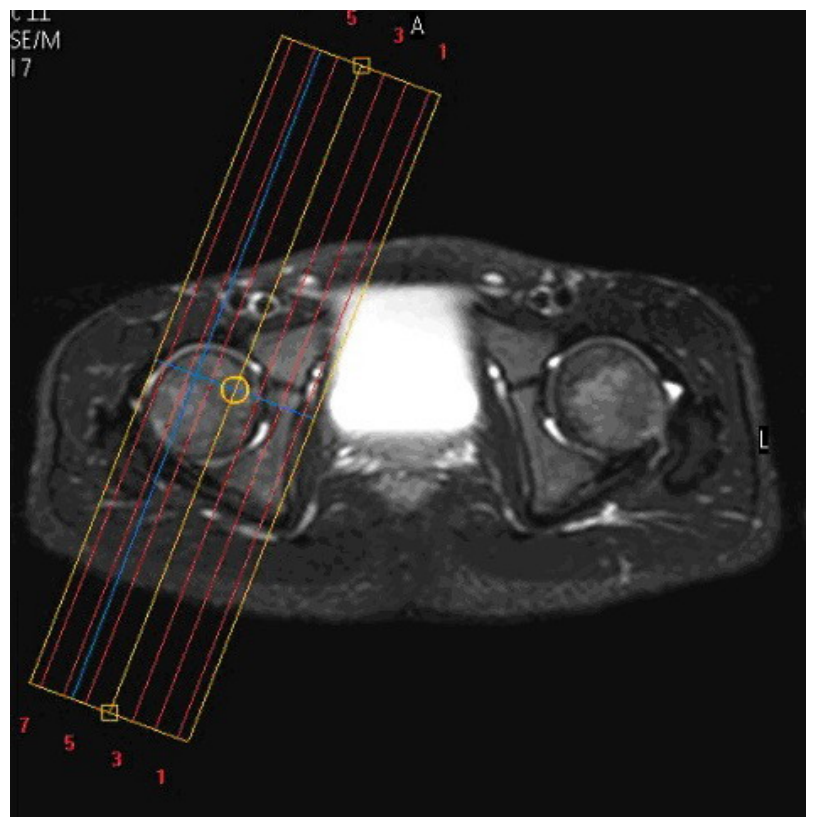

Figure 1. Acetabular anteversion was corrected by putting the orienting line along the margin of acetabulum on axial and coronal directions.

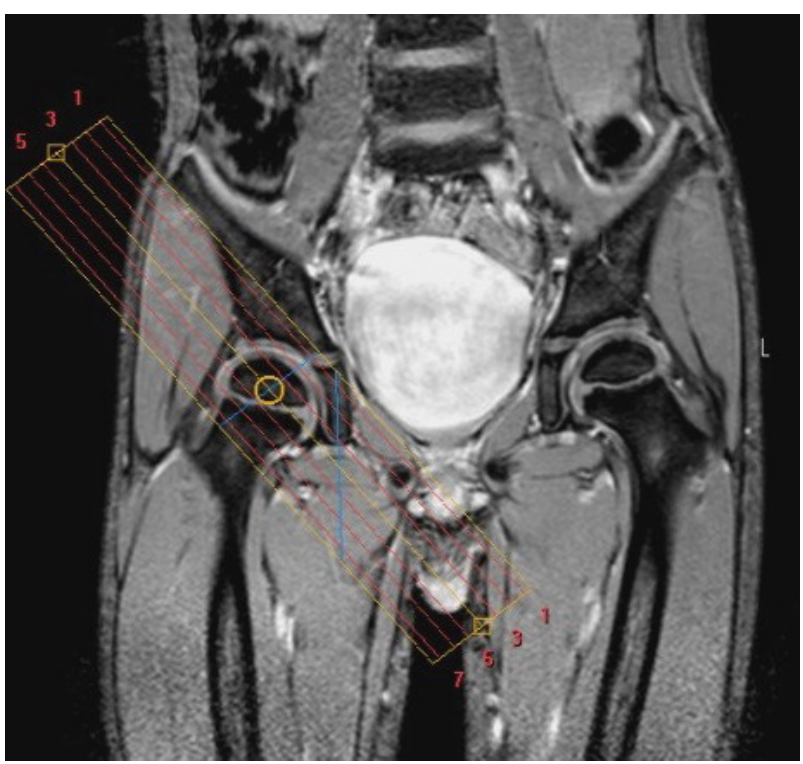

Figure 2. Acetabular anteversion was corrected by putting the orienting line along the margin of acetabulum on axial and coronal directions.

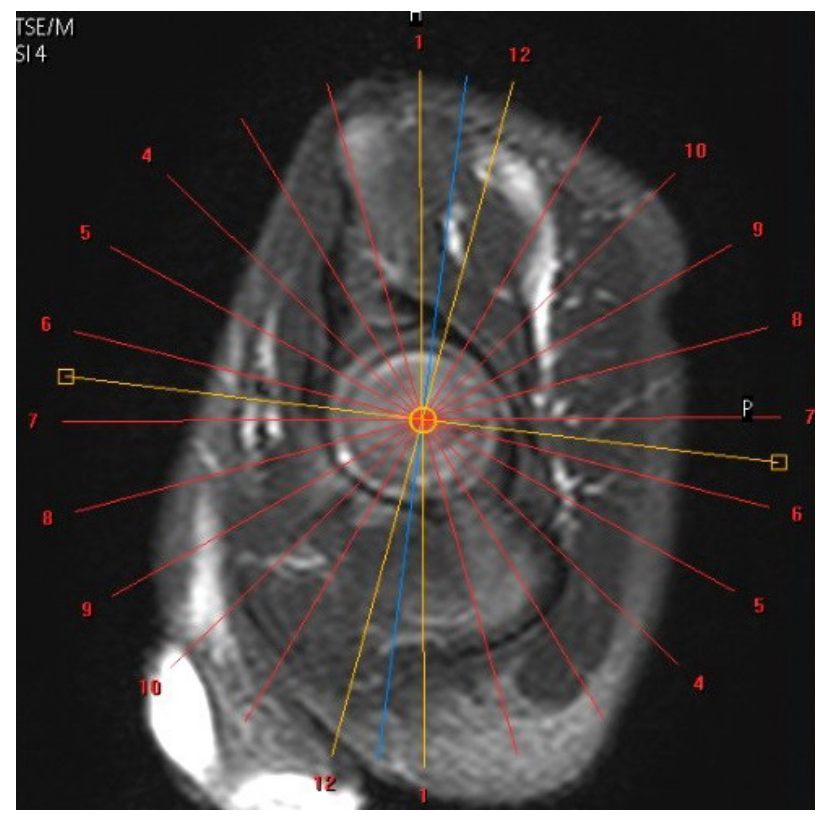

Figure 3. Oblique image of the acetabulum was generated after correction.

Table 1. MR scanning protocol.

\begin{tabular}{lcccc}
\hline & $\begin{array}{c}\text { axial } \\
\text { FS-T2WI }\end{array}$ & $\begin{array}{c}\text { coronal } \\
\text { FFE }\end{array}$ & $\begin{array}{c}\text { oblique } \\
\text { FS-T2WI }\end{array}$ & $\begin{array}{c}\text { radial } \\
\text { FFE }\end{array}$ \\
\hline FOV $(\mathrm{mm} \times \mathrm{mm})$ & $30 \times 30$ & $27 \times 27$ & $25 \times 25$ & $15 \times 15$ \\
$\begin{array}{l}\text { Slice thickness } \\
(\mathrm{mm})\end{array}$ & 4 & 3 & 4 & 3 \\
TR $(\mathrm{ms})$ & 2200 & 440 & 2200 & 300 \\
TE $(\mathrm{ms})$ & 30 & 13.8 & 30 & 13.8 \\
ETL & 10 & 1 & 10 & 1 \\
\hline
\end{tabular}

Note: FS-T2WI: fat-suppressed T2-weighted sequence. 
$15^{\circ}$, mid-superior $0^{\circ}$, and posterosuperior $15^{\circ}, 30^{\circ}$ from anterior to posterior (Figure 4). Total covering angle (TCA), acetabular covering angle (ACA) and labral covering angle (LCA) were measured in each position (Figure 5).

The statistical software used for analysis was SPSS

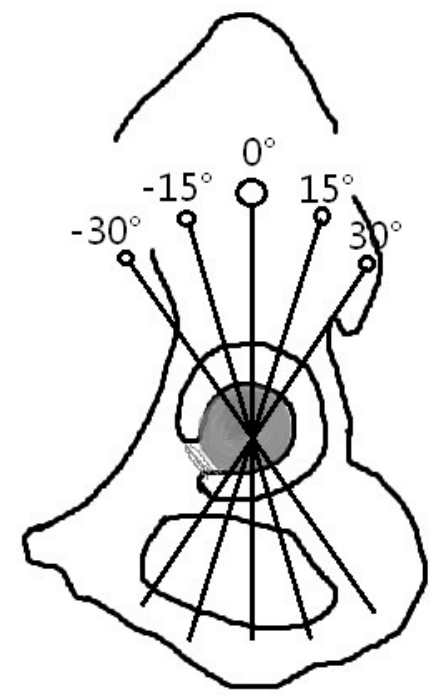

Figure 4. Sketch of 5 sections of the acetabulum at $15^{\circ}$ intervals on center of femoral head from anterosuperior to posterosuperior.

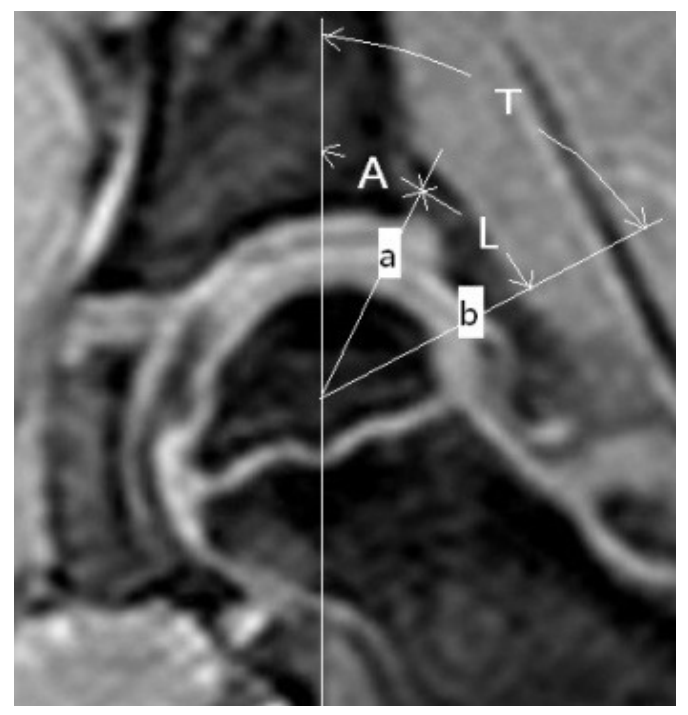

Figure 5. Coverage of femoral head by acetabulum was constituted by three lines: 1) a perpendicular line through the center of the femoral head; 2) a second line connecting the center of the femoral head with the outer margin of the acetabulum (line a); 3 ) a third line connecting the center of the femoral head with the outer margin of the labrum (line b). The angle formed by the perpendicular line and "line a" shows the acetabular covering angle (ACA), the angle formed by the perpendicular line and "line b" shows the total covering angle (TCA), and the angle formed by "lines $a$ and $b$ " shows the labral covering angle (LCA).
16.0 for Windows. Mann-Whitney-U test was used for intergroup comparisons at each position. Spearman's ran$\mathrm{k}$ correlation for the correlation coefficients was used to analyze the relationship between TCA, ACA and LCA. $P$ $<0.05$ was considered significant.

\section{Results}

Congruence of articulating surface of the hip joint at each weight-bearing position was observed in all cases. Margin of bony acetabulum was hard to identify for irregular ossification at the rim in some cases. Of 190 hip joints, clear margin of bony acetabulum was seen in 172 hip joints $(90.53 \%)$. Cartilage surface of the joints was clearer with regular shape. Clear margin of cartilage acetabulum was seen in 188 hip joints $(98.95 \%)$. Acetabular labrum was clearly identified in 180 hip joints $(94.74 \%)$. Of all cases, clear display of anatomical structures including margin of both bony and cartilage acetabulum and the labrum were shown in 158 hip joints $(83.16 \%)$.

There was no statistical TCA difference at every position $(P>0.05)$. There were significant differences of ACA and LCA at posterosuperior $15^{\circ}$, posterosuperior $30^{\circ}$ with other positions $(P<0.05)$. And there were significant differences of ACA and LCA at anterosuperior $30^{\circ}$, anterosuperior $15^{\circ}$, mid-superior $0^{\circ}$ with posterosuperior $15^{\circ}$, posterosuperior $30^{\circ}$ positions $(P<0.05)$. From anterior to posterior of the weight-bearing interface of the hip joints, average ACA increased, while average LCA decreased from anterior to posterior (Table 2). TCA correlated with LCA, but not with ACA. ACA negatively correlated with LCA (Table 3).

\section{Discussion}

The hip joint is the largest weight-bearing joint of congruent ball-and-socket articulation with acetabulum and femoral head. Acetabular cartilage deepens the acetabular fossa by cup-shaped rim of the acetabulum. The labrum is a fibrocartilaginous structure that is firmly attached to outer rim of the acetabulum. The growth of a child's hip from the embryo to adolescence is a sequential process. Growth in depth and construction of the final acetabular shape heavily depends on the interaction with a spherical femoral head [7].

Radiography and computed-tomography have long been used in imaging the hip joint. However, there exists a pitfall of radiographic imaging in visualizing cartilage. MR provides better muscular-skeletal resolution. FFE is a gradient echo sequence widely used in imaging articular cartilage [8]. There have been several MR studies on development of the hip joint in recent years, and there is a raised interest in imaging the normal shape and pathology of acetabular labrum [4-6,9]. A study by DongMei Sun analyzed the features of normal acetabular 
Table 2. Values of TCA, ACA and LCA $(\bar{x} \pm s)$.

\begin{tabular}{ccccc}
\hline & & Anterosuperior & Midsuperior & Posterosuperior \\
\hline Group & $-30^{\circ}$ & $-15^{\circ}$ & $0^{\circ}$ & $30^{\circ}$ \\
TCA & $70.95 \pm 4.34$ & $71.53 \pm 3.82$ & $71.68 \pm 3.56$ & $71.68 \pm 6.46$ \\
ACA & $29.32 \pm 11.00^{\Delta}$ & $28.63 \pm 9.35^{\Delta}$ & $26.47 \pm 8.90^{\Delta}$ & $19.05 \pm 12.08^{*}$ \\
LCA & $42.16 \pm 9.86^{\Delta}$ & $42.47 \pm 9.42^{\Delta}$ & $45.11 \pm 8.67^{\Delta}$ & $52.63 \pm 9.82^{*}$ \\
\hline
\end{tabular}

Note: Intergroup comparisons: ${ }^{*} \mathrm{P}<0.05$ : with anterosuperior $30^{\circ}$, anterosuperior $15^{\circ}$, mid-superior $0^{\circ}$ positions; ${ }^{\#} \mathrm{P}<0.05$ : with posterosuperior $15^{\circ}$ position; $\Delta P<0.05$ : with posterosuperior $15^{\circ}$ and posterosuperior $30^{\circ}$ positions.

Table 3. Correlation coefficients of TCA, ACA and LCA.

\begin{tabular}{cccc}
\hline & TCA-ACA & TCA-LCA & ACA-LCA \\
\hline r-value & 0.515 & $-0.146^{*}$ & $-0.916^{*}$ \\
\hline
\end{tabular}

Note: ${ }^{*} P<0.05$.

labrum and its variation in children. MR appearances of normal acetabular labrum were classified into 3 types: traingle, flat-shape and absent in shape. Triangle shape was most commonly observed and has an increase tendency in development [9]. Proper identification of the labrum could contribute in accurate evaluation of the development of the hip joint. In our study, acetabular labrum was clearly identified in $94.74 \%$ of the cases. Those with unclear shape were mostly located at the posterosuperior position, which may be attributed by imamture development. Margin of the bony acetabulum was hard to identify in some cases for irregular ossification. There is similar trouble when we measure acetabular angle (AA) in radiography, which result in inaccurate quantitative evaluation [10].

Base on the ball-and-socket shape the hip joint, radial scanning through the centre of femoral head can better reflect the physiological status of the joint. With sequential display of the weight-bearing interface from anterior to posterior, coverage of acetabulum to femoral head can be analyzed and measured quantitatively [4-6]. Radial scanning of hip joints were more performed in adults in recent studies. Weight-bearing contribution of bony and cartilageous acetabulum can be evaluated quantitatively by measuring the coverage angle. To the best of our knowledge, radial scanning has not been used in analyzing normal development in child's hip.

TCA, ACA and LCA can reflect the weight-bearing contribution of total acetabulum, bonely acetabulum and the labrum by respective covering proportion. A prior study in our hospital investigated stability of hip joint in normal children from after birth to 12 years old by MR, and found TCA was stable in children after 1 year old, which stand as excellent indicator of articular stability [11]. For the same reason, children under 1 year old were not included in this study for unsure stability. Measurement of coverage angle was confined to superior quarter of the acetabulum from anterosuperior $30^{\circ}$ to posterosuperior $30^{\circ}$ for unclear margin of bony acetabulum and unclear shape of labrum at outer positions. Statistical analysis showed that TCA is stable at every weight-bearing position, whereas there were significant differences of ACA and LCA at some positions. Average ACA increased, while average LCA decreased from anterior to posterior. TCA correlated with LCA, while ACA negatively correlated with LCA. We proposed an idea that total coverage of acetabulum to femoral head at every weight-bearing position remains stable during skeletal maturation. Cartilage ossifies slower at posterior than anterior positions. Cartilage acetabulum and the labrum serve as complementary structures that contribute in total stabilizing of the hip joint in development.

A geometrical analysis of normal development of the hip by planimetric radiography indicated development of acetabulum and femoral head finished at about 10 years old [1]. However, there is always uncertainty in measureing bony parameters for unsure margin of the bony acetabulum [10]. In our study, congruence of articulating is seen at every position in the normal hip joint during development. Measurement of cartilageous structures has better reflection of weigh-bearing stability of the joint. Our findings in a prior study proposed the idea that total coverage of acetabulum to femoral head remains stable during development [11]. Further investigation into every weight-bearing position in this study had findings of stable total coverage angle although with unsynchronized ossification at each position. ACA remains as a good index in indicating the hip joint's development and stability in weight-bearing.

The shortcoming of our study is the limited cases that hinder intergroup analysis into different ages. More cases needed to be collected to verify its significance in children of different ages. Radial scanning can be applied in analyzing overage of femoral head at weight-bearing interface of the hip joint in children. ACA at each weight-bearing position has reflection of the stability of the hip joint in development.

\section{REFERENCES}

[1] C. Birkenmaier, G. Jorysz, V. Jansson, et al., "Normal 
Development of the Hip: A Geometrical Analysis Based on Planimetric Radiography," Journal of Pediatric Orthopaedics, Vol. 19, No. 1, 2010, pp. 1-8.

[2] S. Tamura, T. Nishii, T. Shiomi, et al., "Three-Dimensional Patterns of Early Acetabular Cartilage Damage in Hip Dysplasia; A High-Resolutional CT Arthrography Study," Osteoarthritis and Cartilage, Vol. 20, No. 7, 2012, pp. 642-652.

[3] P. Than, T. Sillinger, J. Kranicz, et al., "Radiographic Parameters of the Hip Joint from Birth to Adolescence," Pediatric Radiology, Vol. 34, No. 3, 2004, pp. 237-244. doi:10.1007/s00247-003-1119-y

[4] Motoyuki Horii, Toshikazu Kubo, Shigehiro lnoue, et al., "Coverage of the Femoral Head by the Acetabular Labrum in Dysplastic Hips: Quantitative Analysis with Radial MR Imaging," Acta Orthopaedica, Vol. 74, No. 3, 2003, pp. 287-292. doi:10.1080/00016470308540842

[5] C. Zilkens, F. Miese, M. Jäger, et al., "Magnetic Resonance Imaging of Hip Joint Cartilage and Labrum," Orthopedic Reviews, Vol. 3, No. 2, 2011, p. e9. doi:10.4081/2641

[6] K. Ueshimaa, K. A. Takahashia, M. Fujiokaa, et al., "Relationship between Acetabular Labrum Evaluation by Using Radial Magnetic Resonance Imaging and Progressive
Joint Space Narrowing in Mild Hip Dysplasia," Magnetic Resonance Imaging, Vol. 24, No. 5, 2006, pp. 645-650. doi:10.1016/j.mri.2005.12.009

[7] M. C. Lee and C. P. Eberson, "Growth and Development of the Child's Hip," Orthopedic Clinics of North America, Vol. 37, No. 2, 2006, pp. 119-132.

[8] M. J. Graves, S. Wakely, P. W. Bearcroft, et al., "MRGuided Direct Arthrography of the Hip," Journal of Magnetic Resonance Imaging, Vol. 8, No. 2, 2008, pp. 462465. doi:10.1002/jmri.21432

[9] D.-M. Sun, S.-N. Pan, Z.-M. Lu, et al., "3.0T MRI Features of Normal Acetabular Labrum in Children," Chinese Journal of Medical Imaging Technology, Vol. 26, No. 3, 2010, pp. 532-534.

[10] A. N. Larson, B. Tabenhorst, A. De La Rocha, et al., "Limited Intraobserver and Interobserver Reliability for the Common Measures of Hip Joint Congruency Used in Dysplasia," Clinical Orthopaedics and Related Research, Vol. 470, No. 5, 2012, pp. 1414-1420. doi:10.1007/s11999-011-2136-Z

[11] S.-H. Zeng, H.-S. Liu, H.-W. Xu, et al., "Quantitative MRI Analysis of Normal Development of the Hip in Children," Chinese Journal of Medical Imaging Technology. Vol. 27, No. 7, 2011, pp. 40-44. 